\title{
VARIATION, STYLE ET NORME : HÉRITAGE PRAGOIS ET ÉLABORATIONS ULTÉRIEURES
}

\author{
Sándor KISS \\ Université de Debrecen
}

\begin{abstract}
En): The approach to "norm" is attempted here within the frame of linguistic variation and in connection with stylistic phenomena resulting from the connotative meaning of signs. The language speakers, through their choices among offered possibilities, intuitively create linguistic standards. In a period of normalization, one of the standards is preferred and becomes decisive in usage. Linguists of the Prague School have already founded the theory of norms, and sought consciously to lay out the prerequisites for the efficiency of linguistic communication in the modern world.
\end{abstract}

Keywords (En): Norm; linguistic variation; connotation; Prague School

Mots-clés (Fr): Norme ; Variation linguistique ; Connotation ; École de Prague

\section{Introduction}

La notion de norme, point de départ fécond de toute une réflexion approfondie des linguistes pragois, conduit assez naturellement vers d'autres concepts qui éclairent son essence et permettent de la situer parmi les données fondamentales de l'usage : c'est ce que reflète le titre de ma contribution. En effet, la norme ensemble d'habitudes observées par les membres d'une communauté linguistique (habitudes formées en partie spontanément et répondant en partie à des prescriptions) - a pour cadre la variation de l'usage, car une norme existe nécessairement par rapport à d'autres variantes linguistiques. D'autre part, en ce qui concerne le style, celui-ci est souvent perçu comme un ensemble de choix qui oscillent entre deux pôles: celui de la «neutralité » (synonyme ici de «normalité ») et celui de la «spécificité » (qui signifie un écart par rapport à la norme).

\section{Style et connotation}

Néanmoins, le phénomène du style peut être abordé sous un autre angle d'une manière moins restrictive, pourrait-on dire. Pour définir cette notion plus compréhensive du style, je m'appuierai sur la distinction bien connue entre le concept de «dénotation» et celui de «connotation». Il me semble que si l'on donne une interprétation assez large du terme de «connotation », en l'appliquant aux signes et aux combinaisons de signes, on obtient une clé qui permet de saisir l'ensemble des phénomènes stylistiques. Il faut d'abord insister sur la nécessité de la connotation : en dehors de leur sens référentiel (dénotatif) ou opérationnel (par exemple : conjonctif), tous les signes transmettent, dans la communication, un ensemble de significations secondaires (connotatives), qui proviennent de deux types de sources : elles ont une origine soit intralinguistique, soit extralinguistique. On peut appeler sources intralinguistiques de la connotation toutes les propriétés 
du signe qui se laissent déduire d'une comparaison avec les autres signes de la langue, comme l'appartenance à telle ou telle partie du discours, à un schéma phonologique habituel ou atypique, le caractère simple ou complexe de l'expression, la visée directe ou périphrastique, la place dans une série synonymique, la fréquence relative. En revanche, les sources extralinguistiques de la connotation se constituent grâce au «trafic » du signe dans le monde, pour ainsi dire : un mot, une forme grammaticale, un tour syntaxique sont marqués par leurs conditions d'emploi, ils se rattachent, dans la mémoire des usagers, à un milieu, à un métier, à une relation humaine particulière, le signe possède donc une puissance d'évocation sociale; mais tous les mots, toutes les combinaisons de mots, toutes les structures ont en même temps une dimension iconique, ils produisent des impressions sensuelles, «matérielles », ils imitent, ils représentent par ressemblance : ce sont des choses parmi les choses du monde. L'ensemble des connotations, qui accompagnent ainsi nécessairement la dénotation, constitue un message secondaire, que nous pouvons appeler le message stylistique.

En ce qui concerne maintenant les sources internes de la connotation, que nous pouvons aborder par le biais de la comparaison entre signes, cet aspect du style est entièrement déductible du principe saussurien de la valeur. SAUSSURE (1922/1972 : 160) dit ceci : «Dans l'intérieur d'une même langue, tous les mots qui expriment des idées voisines se limitent réciproquement: des synonymes comme redouter, craindre, avoir peur n'ont de valeur propre que par leur opposition; si redouter n'existait pas, tout son contenu irait à ses concurrents ». La constatation de Saussure incite à chercher la différence entre termes voisins non seulement sur le plan notionnel, mais aussi du point de vue de leur structure interne, de leur fréquence et de leur accessibilité mémorielle. Cette exigence est bien claire pour le structuralisme européen. Citons l'un des fondateurs de l'École de Prague, Roman Jakobson, qui a explicité cette problématique au cours des remarques qu'il a faites sur la traduction. La «traduction intralinguale», dit-il (JAKOBSON, 1959/1963: 79-80), habituellement une périphrase, offre une expression plus transparente, mais sans doute moins usuelle de la notion en question : le terme complexe personne non mariée,pour célibataire, est le produit d'une explicitation et relève de l'activité interprétative. Selon le même auteur, des tests ont montré la fonction connotative du genre grammatical (catégorie qui peut avoir pourtant une signification dénotative): des locuteurs russes, invités à personnifier les jours de la semaine, les représentaient comme des êtres soit masculins, soit féminins, suivant le genre des termes (понедельник 'lundi', вторник 'mardi', четверг 'jeudi' masculins, face à среда 'mercredi', пятница 'vendredi', суббота 'samedi' féminins, op. cit. : 84). Quant aux sources externes de la connotation, les linguistes de l'École de Prague ont insisté sur les phénomènes de la parole individuelle, sur la « linguistique de la parole ${ }^{1}$. C'est ce que fait Troubetzkoy lorsqu'en fondant la discipline linguistique appelée phonologie expressive ou phonostylistique, il se sert de l'exemple des allongements vocaliques et consonantiques en allemand. La durée des différents types de sons dépend naturellement, en premier lieu, de la structure d'une langue

\footnotetext{
${ }^{1}$ Pour une approche théorique, cf. SKALIČKA (1948/1964).
} 
donnée, mais, ayant un aspect symbolique plus général, elle peut ajouter un message secondaire au message proprement référentiel. Le mot schön peut être prononcé avec «ravissement»: schschöön! 'admirable!', «mais aussi avec ironie »; schschaamlos 'impudent' «avec indignation»; lliieber Freund 'cher ami' " avec enthousiasme, avec ironie, avec indignation, sur un ton persuasif, avec regret ou pitié, etc., et chaque fois avec une autre nuance d'intonation» (TROUBETZKOY, 1939/1949 : 25). Le symbolisme universel de certains procédés phonétiques peut se doubler d'une signification sociale : «Dans les communautés linguistiques fortement différenciées, ces distinctions [phonostylistiques] sont très marquées dans les prononciations qui reposent sur une structure provinciale, professionnelle ou culturelle de la société » (op. cit.: 21). J. MUKAŘOVSKÝ(1932/1964: 17) décrit cette problématique du point de vue de l'usage poétique, en citant notamment des archaïsmes utilisés dans la langue littéraire tchèque.

\section{Pluralité des normes}

Une certitude est acquise : dans le processus de la communication linguistique, tous les aspects du discours sont interprétés par les décodeurs. Ainsi, les locuteurs, qui acquièrent une impression confuse de leur propre manière de parler, sont instinctivement amenés à choisir entre les variantes disponibles et à établir des distinctions sémantiques, en fixant ou en déplaçant la valeur d'un signe selon les besoins de la communication et en réglant leur comportement linguistique par rapport à des normes changeantes ${ }^{2}$. Que la norme ne soit jamais entièrement une et stable ne l'empêche nullement d'exister et de régler les manifestations linguistiques : les locuteurs s'adaptent, d'une manière plus ou moins inconsciente, à des habitudes dominantes, qui permettent cependant des écarts. En fait, les normes peuvent être multiples au sein d'une communauté linguistique, et tous les locuteurs individuels n'ont pas à leur portée le même ensemble de normes. C'est ainsi que les sujets parlants qui, par leur éducation, ont accès à un code relativement étendu peuvent connaître des codes plus restreints, des normes moins complexes et s'en servir au besoin. Cicéron a fait remarquer qu'il ne se sentait pas tenu d'utiliser un langage distingué dans les situations qui n'avaient rien de solennel ${ }^{3}$. En guise de parallèle, nous pouvons citer l'entrée «Style et stylistique » de La linguistique - guide alphabétique, petite encyclopédie rédigée par A. MARTINET (1969: 360), à propos du «laisser-aller» que se permettent les locuteurs français, « même instruits », par rapport à la prescription académique, en disant je peux pas, je m'en rappelle, y a qu'à, pour je ne peux pas, je m'en souviens, il n'y a qu'à. En évoquant la norme ancienne du français cultivé, les

\footnotetext{
${ }^{2}$ Comme l'a souligné B. HAVRÁNEK (1936/1964 : 414), la norme n'est pas un phénomène qui soit propre à la seule langue écrite : les membres de toutes les communautés linguistiques suivent dans leur usage un standard qui leur est prescrit par la coexistence sociale elle-même. L'auteur explicite ensuite les facteurs dont le jeu conjugué peut transformer ce standard (op. cit. : 417).

3 «ipsa iudicia non solemus omnia tractare uno modo [...] epistulas uero cotidianis uerbis texere solemus " 'même les affaires judiciaires, nous ne les traitons pas [linguistiquement] sur le même mode $[\ldots]$, et en ce qui concerne les lettres, nous les tissons habituellement avec des mots quotidiens' (Cicéron, Epistulae ad familiares 9,21,1).
} 
auteurs ajoutent: «si nous nous en tenons aux règles de la grammaire traditionnelle, force nous sera de considérer comme faisant partie de la norme une construction aussi désuète que il serait bon que j'y allasse, alors que l'imparfait du subjonctif est, précisément de nos jours, un procédé stylistique, voire comique, des plus flagrants $\gg$.

\section{Standardisation}

Le heurt entre sociolectes - ou éventuellement entre dialectes qui peuvent être porteurs eux-mêmes d'un haut prestige social - devient clairement perceptible lorsque dans la société se fait sentir un besoin de réglementation, dans le but d'élaborer la variante standardisée d'un idiome. À une période charnière de l'évolution du français, Molière mène un combat linguistique sur deux fronts : il ridiculise le parler des gens sans instruction ${ }^{5}$, et il attaque ceux - ou plutôt celles qui lui semblent compromettre l'efficacité du message par excès de finesse et d'analyse $^{6}$. Malgré certaines injustices de sa critique, on peut dire que Molière a compris une nécessité de l'époque : le «besoin de sagesse dans la langue », selon la formulation de Ferdinand BRUNOT (1922: 2). C'est le besoin d'une communication ordonnée et efficace à un moment où la vie des élites citadines devient de plus en plus complexe. Les règles du contact entre individus sont élaborées avec précision, et l'importance du texte écrit ne cesse d'augmenter dans l'existence quotidienne. Il n'est pas difficile de réduire à sa juste valeur la fameuse boutade de Malherbe, qui s'en remettait, disait-il, aux crocheteurs du Port-au-Foin en matière de correction linguistique : Malherbe veut la simplicité et la précision, sans la vulgarité. BRUNOT (op. cit. : 6) commente judicieusement : "Malherbe n'admettait pas qu'on pût écrire un mot que les crocheteurs ne comprissent et ne connussent pas; mais [...] loin d'accepter en bloc dans sa crudité ce parler du Port-au-Foin, l'écrivain devait choisir, et se montrer très scrupuleux dans son triage ». Bien entendu, il ne s'agit pas uniquement de créer un standard compréhensible et, pour ainsi dire, démocratique; en fait, les prescriptions «classiques» saisissent et érigent en norme ce qui a été préparé «depuis

\footnotetext{
${ }^{4}$ HAVRÁNEK (op. cit. : 414) a déjà nettement distingué la codification de la norme et la norme effectivement suivie par les locuteurs.

${ }^{5}$ Citons, en guise d'illustration, la scène I de l'acte II de Dom Juan : «CHARLOTTE : Ne t'aimé-je pas aussi comme il faut ? PIERROT : Non : quand ça est, ça se voit, et l'en fait mille petites singeries aux personnes quand on les aime du bon du cœur. Regarde la grosse Thomasse, comme elle est assotée du jeune Robain : alle est toujou autour de li à l'agacer, et ne le laisse jamais en repos ; toujou al li fait queuque niche ou li baille queuque taloche en passant », etc.

${ }^{6}$ Les Précieuses ridicules, scène IV : «CATHOS : En effet, mon oncle, ma cousine donne dans le vrai de la chose. Le moyen de bien recevoir des gens qui sont tout à fait incongrus en galanterie ? Je m'en vais gager qu'ils n'ont jamais vu la carte de Tendre, et que Billets-Doux, Petits-Soins, BilletsGalants et Jolis-Vers sont des terres inconnues pour eux. Ne voyez-vous pas que toute leur personne marque cela, et qu'ils n'ont point cet air qui donne d'abord bonne opinion des gens ? Venir en visite amoureuse avec une jambe toute unie, un chapeau désarmé de plumes, une tête irrégulière en cheveux, et un habit qui souffre une indigence de rubans ! » Le langage des précieuses constitue pour Molière un objet de raillerie à cause de sa tendance à l'abstraction et à la métaphorisation, recherche pourtant naturelle à un moment où les questions du langage acquièrent dans la vie intellectuelle une importance primordiale.
} 
longtemps par l'évolution de la langue»(BRUNOT, ibid.) : pas et point sont déclarés obligatoires en tant que seconds éléments de la négation; le pronom sujet du verbe ne doit plus être omis. Cependant, les «législateurs» classiques se chargent également de recréer le standard littéraire, en faisant triompher un certain goût, préconisé par les élites, où la richesse de l'expression linguistique et les audaces de l'imagination sont soumises à la règle de l'équilibre?. Malherbe poète contribue à tracer le chemin de cette nouvelle esthétique disciplinée :

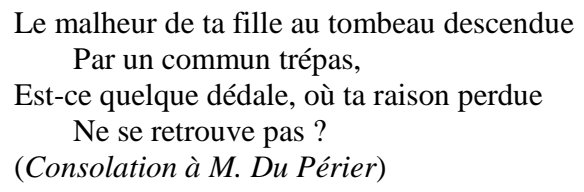

On est tout de même loin de la vraie simplicité et du stilus humilis.

\section{Recherche pragoise de la «stabilité flexible »}

Étant parti de la notion de norme et faisant un détour vers la variation, que j'ai illustrée par l'omniprésence des valeurs stylistiques, me voilà donc revenu au problème de la communication «normalisée » ou «standardisée », problème à résoudre dans la société pour assurer un contact linguistique exigeant et aussi pour forger une image de marque de la communauté. Les chercheurs de l'École de Prague, engagés dans l'entreprise de renouveler le standard de la langue tchèque cultivée et insistant sur l'importance de la culture de la langue, sont conscients de se charger d'une tâche délicate, comme cela apparaît dans leurs différentes prises de position concernant la langue des intellectuels et la langue littéraire ${ }^{8}$.

En fait, l'entreprise de ces linguistes est extrêmement originale: nous saisissons là un des rares moments où la science du langage entre dans un contact direct avec la pratique ordinaire de la langue, dont elle est capable d'influencer l'usage courant. L'activité standardisante des linguistes pragois, qui visait à la fois la stabilisation et l'enrichissement de la langue tchèque ${ }^{9}$, faisait suite aux efforts de renouvellement culturel et linguistique de la Bohême, remontant à l'époque des Lumières, et dont le but était de créer une culture tchèque moderne, avec une langue nationale basée sur la tradition, mais capable de satisfaire les exigences d'une vie intellectuelle - philosophique, juridique, littéraire - rapidement épanouie $^{10}$.

La tâche des linguistes pragois n'était pas facile. En effet, ils devaient éviter deux pièges: celui du purisme et celui de l'innovation désordonnée. Une

\footnotetext{
${ }^{7}$ Cette réglementation correspond, bien entendu, à l'esprit général de l'époque, « toute de centralisation et d'autorité » (BRUNOT, $1911: 74)$.

${ }^{8}$ Cf. notamment les «Thèses générales du Cercle Linguistique de Prague : Principes pour la culture de la langue » (1932), traduites et réimprimées dans BÉDARD - MAURAIS (1983 : 799-807).

${ }^{9}$ Les travaux linguistiques théoriques doivent contribuer « $1^{\circ}$ à la stabilité de la langue standard et $2^{\circ}$ à sa capacité de différenciation fonctionnelle et à son enrichissemnt stylistique » («Thèses générales », dans BÉDARD - MAURAIS, 1983 : 799).

${ }_{10}$ Concernant les conditions externes de l'évolution de la langue tchèque moderne, v. GARviN (1983: 141-144).
} 
communauté linguistique qui cherche à renouveler la langue défend instinctivement son patrimoine contre les influences étrangères. Dans le cas de la langue tchèque, c'était la pénétration du lexique allemand qui apparaissait comme un danger ${ }^{11}$; il est compréhensible, d'autre part, que les besoins de la civilisation moderne aient fait naitre un bon nombre d'innovations, surtout lexicales, qui heurtaient le goût traditionaliste. On constate cependant que d'éminents linguistes de l'École de Prague ont su trouver, dès le début de l'activité du groupe, le juste milieu au cours de ces débats. Roman Jakobson a évoqué notamment les effets bienveillants de l' «hybridation », subie par les grandes langues de civilisation, tout en reprochant aux puristes de ne pas tenir compte de la «nécessité vitale » de

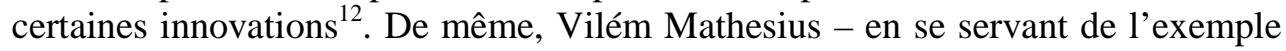
de l'anglais et des langues romanes - explique qu'il n'y a pas de «relation de cause à effet entre la pureté historique et un haut niveau de développement linguistique $»^{13}$.

En fin de compte, le principal problème qui s'est posé aux linguistes pragois en matière de « législation » linguistique consistait à concilier une relative unité de la langue soignée avec la sauvegarde de la créativité - donc avec ce qu'ils ont appelé « la différenciation fonctionnelle et [...] l'enrichissement stylistique de la langue standard» («Thèses générales », BÉDARD - MAURAIS, 1983 : 806). Pour formuler cette exigence, nécessairement synthétique, Vilém MATHESIUS $(1932 / 1983: 811)$ a trouvé l'heureuse expression de «stabilité flexible». Selon les «Thèses générales » (p. 800), la norme de la langue standard cultivée doit se fonder «sur la pratique littéraire moyenne des cinquante dernières années », en écartant tout ce qui représenterait une particularité insolite par rapport à l'usage courant (éléments populaires, dialectaux, argotiques, archaïques, expressions littéraires difficilement compréhensibles); mais, d'autre part, selon les mêmes « Thèses » (p. 805), le « vocabulaire standard » ne doit pas « être limité à ce que la norme a englobé jusqu'ici » : il doit satisfaire aux besoins de la communauté, en permettant la pénétration des néologismes. Le travail réalisé par ces linguistes porte ainsi l'empreinte du fonctionnalisme pragois. C'est le même sens de l'équilibre qui se manifeste dans les développements consacrés à la réglementation syntaxique : «la codification ne doit pas cultiver inutilement l'archaïsme», cependant, elle ne doit pas non plus «empêcher la création des mécanismes syntaxiques spéciaux par lesquels la langue littéraire diffère du discours familier » («Thèses générales »: 805). Selon la formulation de MATHESIUS (1932/1983: 813), la tâche du linguiste est «de codifier l'usage soigné d'aujourd'hui et de limiter les variations qui ne sont pas fonctionnellement justifiables ».

Comme le remarquent les auteurs des «Thèses » (p. 802), les interventions des linguistes ne doivent pas chercher à éliminer de la langue tous les flottements, car ceux-ci garantissent « la diversité fonctionnelle et stylistique nécessaire à la langue

\footnotetext{
${ }^{11}$ GARVIN (1983: 142).

${ }^{12}$ Pour les idées de Jakobson concernant le purisme et l'innovation linguistiques, cf. GARvin, 1983 : 142-143. Pour la coexistence et la lutte de ces différentes tendances dans l'usage linguistique et notamment dans la langue littéraire, v. HAVRÁNEK (op. cit. : 418).

13 «Sur la nécessité de stabilité d'une langue standard » (1932), article traduit et réimprimé dans BÉDARD - MAURAIS (op. cit. : 810).
} 
standard ». C'est la communication effective qui est visée, avec un trait original qui affleure dans tous les projets : notamment l'exigence d' « intellectualisation » de la langue standard, prescrivant l'emploi précis des vocables et prévoyant, au besoin, la création de termes abstraits, «capables d'exprimer la "connectivité" et la complexité de la pensée $»^{14}$. La recherche de la norme s'enrichit par une dimension sociologique : «la norme d'une langue standard est plus consciente et plus contraignante que celle d'une langue populaire, et la nécessité de la stabilité, plus prononcée $\gg$. MATHESIUS (1932/1983: 811-812) a remarqué : «l'élaboration d'une conscience linguistique pour la nouvelle langue standard tchèque » était une entreprise nécessaire et efficace.

\section{Conclusion : standard et variétés}

La variation, considérée aujourd'hui par la sociolinguistique comme une propriété inhérente à la réalisation du système linguistique, apparaît ainsi, dès les travaux des membres de l'École de Prague, comme un fait à décrire scientifiquement. En même temps, la variation constitue pour eux une virtualité, dont l'exploitation est indispensable pour forger un outil de communication de haute valeur. Cette prise de conscience des linguistes pragois se retrouvera par la suite dans de nombreuses cultures, dont l'élite intellectuelle devra affronter des problèmes de normalisation linguistique au seuil de l'éveil national ${ }^{16}$. On sait d'autre part que la linguistique contemporaine cherche à distinguer, lors de la description d'une langue, les différentes variétés du système, surtout au point de vue sociolinguistique. Selon la formulation radicale de COSERIU, une description structurale doit avoir pour objet une seule variété temporelle, spatiale, sociale et stylistique de la langue en question ${ }^{17}$. Néanmoins - comme l'ont bien vu les linguistes de l'École de Prague aussi - ces variétés n'existent pas indépendamment les unes des autres, au contraire : ce sont leurs rapports mutuels qui, par le biais des innombrables choix des locuteurs, poussent les langues vers le changement, en leur permettant de s'adapter aux besoins de la communication ${ }^{18}$. Naturellement, les types de description qui visent avant tout à construire des modèles de la compétence et à découvrir des universaux du langage derrière les particularités

\footnotetext{
${ }^{14}$ Dans le domaine de la philosophie ou pour le métalangage de la science du langage, par exemple. Cf. Bohuslav HAVRÁNEK : «Emploi et culture de la langue standard » (1932), traduit et réimprimé dans BÉDARD - MAURAIS (1983: 822).

${ }^{15}$ HAVRÁNEK (1932: 816).

${ }^{16}$ La communauté linguistique galicienne, retrouvant peu à peu son identité parmi les idiomes de la péninsule Ibérique, offre ici un exemple caractéristique. Concernant les problèmes généraux de la réforme et du renouvellement lingustiques, cf. FODOR István, HAGÈGE Claude (éds.) (1983-1994), Language Reform - History and Future, Hamburg, Buske.

17 «nicht nur synchronisch [...] sondern [...] auch syntopisch, synstratisch und symphasisch» (COSERIU, 1992: 286; pour l'explication détaillée des variétés diatopiques, diastratiques et diaphasiques, v. ibid. : 280-283).

${ }^{18}$ Dans leur étude fondamentale, proposant une théorie sociolinguistique de la modification des langues, WeINREICH, LABOV et HERZOG (1968: 187-188) arrivent à cette conclusion : «Linguistic structure includes the orderly differentiation of speakers and styles through rules which govern variation in the speech community (...) all change involves variability and heterogeneity ».
} 
offertes par différentes langues mettent l'accent sur l'unité du système et de la communauté linguistique où il est utilisé et non sur leur diversité interne ${ }^{19}$.

Dans l'approche sociolinguistique des langues et de leurs modifications, la notion de standard joue naturellement un rôle fondamental; la naissance des variantes à la fois prestigieuses et utiles doit être décrite en rapport avec les transformations de la communauté linguistique, qui influencent la structure linguistique et redéfinissent sans cesse le réseau des connotations, donc le tableau des virtualités de style. Comme nous l'avons vu, les membres de l'École de Prague avaient une intuition profonde de la variation linguistique, ainsi que du rapport du locuteur avec la langue qu'il parle; en vue d'assurer pour la variante littéraire de la langue tchèque une «stabilité flexible», ils se sont livrés à une analyse minutieuse du passé de cette langue, en plaçant cette recherche dans le cadre d'une investigation culturelle au sens large. Tout compte fait, on peut affirmer que, dans le contexte historique et social où ils travaillaient, leur entreprise, scientifique par sa méthode et par ses résultats de base, répondait aussi à un défi, éveillant en eux un sentiment de responsabilité intellectuelle.

\section{BIBLIOGRAPHIE}

BÉDARD Édith, MAURAIS Jacques (éds.) (1983), La norme linguistique, Québec, Conseil de la Langue Française.

BRUNOT Ferdinand $\left(1922^{2}\right.$ ), Histoire de la langue française III, Paris, Armand Colin.

BRUNOT Ferdinand (1911), Histoire de la langue française IV, Paris, Armand Colin.

ChOMsky Noam (1965/1971), Aspects de la théorie syntaxique, Paris, Seuil (traduction de J.-Cl. Milner).

COSERIU Eugenio $\left(1992^{2}\right)$, Einführung in die allgemeine Sprachwissenschaft, Tübingen, Francke.

GARVIN Paul L. (1983), Le rôle des linguistes de l'École de Prague dans le développement de la norme linguistique tchèque, in: BÉDARD Édith, MAURAIS Jacques (éds.) (1983), La norme linguistique, Québec, Conseil de la Langue Française, p. 141-152.

HAVRÁNEK Bohuslav (1936/1964), Zum Problem der Norm in der heutigen Sprachwissenschaft und Sprachkultur, in: VACHEK Josef (éd.), A Prague School Reader in Linguistics, Bloomington, Indiana University Press, p. 413420.

JAKOBSON Roman (1959/1963), Aspects linguistiques de la traduction, in : Essais de linguistique générale, Paris, Minuit, p. 78-86 (traduction de N. Ruwet).

\footnotetext{
19 «L'objet premier de la théorie linguistique est un locuteur-auditeur idéal, appartenant à une communauté linguistique complètement homogène» (СHOMSKY, 1965/1971: 12). Pour un commentaire de cette attitude caractérisant la grammaire générative, cf. l'étude de MORIN et PARET (1983 : 194) : on partira de l'observation d' ' un petit groupe qui semble avoir les mêmes usages et partager les mêmes jugements. On examinera dans ces usages et ces jugements ce qui semble relever des problèmes de mémoire, d'attention, de l'hétérogénéité de la communauté, etc., pour ne prendre en charge que ce qui est du domaine de la compétence grammaticale».
} 
MARTINET André (éd.) (1969), La linguistique. Guide alphabétique, Paris, Denoël.

MATHESIUS Vilém (1932/1983), Sur la nécessité de stabilité d'une langue standard, in: BÉDARD Édith, MAURAIS Jacques (éds.) (1983), La norme linguistique, Québec, Conseil de la Langue Française, p. 809-813.

MORIN Yves-Charles, PARET Marie-Christine (1983), Norme et grammaire générative, in: BÉDARD Édith, MAURAIS Jacques (éds.) (1983), La norme linguistique, Québec, Conseil de la Langue Française, p. 179-202.

MUKAŘOVSKÝ Jan (1932/1964), Standard Language and Poetic Language, in : GARVIN Paul L. (éd.), A Prague School Reader on Esthetics, Literary Structure, and Style, Washington, Georgetown University Press, p. 17-30 (traduction de P. L. Garvin).

SAUSSURE Ferdinand de (1922/1972), Cours de linguistique générale, Paris, Payot.

SKALIČKA Vladimír (1948/1964), The Need for a Linguistics of «la parole », in : VACHEK Josef (éd.), A Prague School Reader in Linguistics, Bloomington, Indiana University Press, p. 375-390.

TroubetzKoy Nicolaï Serguéievitch (1939/1949), Principes de phonologie, Paris, Klincksieck (traduction de J. Cantineau).

WEINREICH Uriel, LABOV William, HERZOG Marvin I. (1968), Empirical Foundations for a Theory of Language Change, in: LEHMANN W. P., MALKIEL Yakov (éds.), Directions for Historical Linguistics, Austin London, University of Texas Press, p. 95-188. 